\title{
Criterios de predicción de inestabilidades plásticas en procesos de conformado en caliente. (Parte II. Caracterización de la fluencia plástica de un acero microaleado de medio carbono usando criterios continuos y fenomenológicos) $)^{(\cdot)}$
}

\author{
A. Al Omar*, I. Alcelay* y J.M. Prado**
}

\begin{abstract}
Resumen
En el estudio de los procesos de conformado en caliente es muy importante conocer la interacción entre el comportamiento microestructural y los parámetros de control del proceso de deformación, tales como temperatura, velocidad de deformación, deformación, etc. En las últimas décadas, los mapas de procesado han sido elaborados para diseñar, controlar y optimizar la deformación en caliente de varios materiales metálicos. En el presente trabajo, con el fin de estudiar la conformabilidad en caliente de un acero microaleado de medio carbono, durante ensayos de compresión en caliente, se presenta un estudio comparativo entre dos tipos de mapas de procesado construidos usando criterios termodinámicos continuos y criterios fenomenológicos. El análisis de los mapas de procesado indica que el acero estudiado no sufre ningún tipo de inestabilidad plástica. Sin embargo, los mapas correspondientes a la deformación $\varepsilon=0,6$ revelan la existencia de un dominio de recristalización dinámica, considerado como el dominio más eficiente dentro de la región segura de procesado. Este dominio está centrado a $1.150^{\circ} \mathrm{C}$ y $10 \mathrm{~s}^{-1}$. El estudio comparativo de los resultados obtenidos muestra la diferencia entre las posiciones de los distintos dominios de deformación plástica según el criterio de inestabilidad usado.
\end{abstract}

Palabras clave

Conformado en caliente; Acero microaleado; Mapas de inestabilidad plástica; Recristalización dinámica.

\section{Criteria for prediction of plastic instabilities for hot working processes. (Part II. Characterization of plastic flow of medium carbon microalloyed steel using phenomenological and continuum criteria)}

\begin{abstract}
In the study of hot forming processes the knowledge of interaction between microstructural behaviour and control process parameters such as temperature, strain rate and strain is very important. In the last decades, processing maps have been developed to design, control and optimize the hot deformation of various metallic materials. In this work, in order to study the hot formability of medium carbon microalloyed steel, during hot compression tests, a comparative study between two types of processing maps constructed using phenomenological and thermodynamic continuum criteria have been carried out. The analysis of the maps indicates that the studied steel does not undergo any type of plastic instability. However, the maps corresponding to the deformation of $\varepsilon=0,6$ reveal a domain of dynamic recrystallization, considered as the more efficiently domain within the "safe" region process. This domain is centered at $1150^{\circ} \mathrm{C}$ and $10 \mathrm{~s}^{-1}$. Also, the comparative study of the obtained results shows the difference between the positions of plastic deformation domains predicted by the two criteria.
\end{abstract}

Keywords

Hot working; Microalloyed steel; Plastic instabilities maps; Dynamic recrystallization; Flow localization.

\section{INTRODUCCIÓN}

La crisis económica mundial plantea un serio reto a la industria de automoción. La necesidad de una mayor competitividad y reducción de costes y el aumento de la demanda de una energía eficiente y renovable, obligan a todos los sectores tecnológicos a ser muy exigentes en cuanto a la optimización de procesos de

\footnotetext{
(•) Trabajo recibido el día 16 de julio de 2009 y aceptado en su forma final el día 19 de noviembre de 2009.

* Departamento de Ingeniería Mecánica, EPSEM, Universidad Politécnica de Cataluña, Av. Bases de Manresa 61 - 73, 08240 Manresa, Barcelona, España.

${ }^{* *}$ Centro Tecnológico de Manresa, Av. Bases de Manresa 1, 08240 Manresa, Barcelona, España.
} 
fabricación, para poder conseguir unas prestaciones elevadas y una durabilidad suficiente en las piezas fabricadas. En este sentido, la utilización de los aceros microaleados en la fabricación de componentes de automoción ha crecido en las últimas décadas. Su interés se deriva del hecho de no necesitar los costosos tratamientos térmicos de temple y revenido después de la forja de la pieza. Por lo tanto, el empleo de estos aceros, especialmente los de contenido medio de carbono, permite reducir los costes económicos y energéticos de fabricación de piezas forjadas en aproximadamente un 15-25\%, en función del tipo de pieza. En este tipo de aceros, el control de las propiedades y de la microestructura se logra a través del afino de grano y del endurecimiento por precipitación durante el enfriamiento de la ferrita ${ }^{[1-6]}$.

Además, la creciente demanda de productos con formas geométricas cada vez más complicadas dentro de unas tolerancias muy estrechas, la reducción en los tiempos de fabricación así como la reducción en los tiempos de lanzamientos al mercado de los nuevos productos, llevó a muchos investigadores a emprender y realizar varios trabajos de investigación para mejorar y optimizar los procesos de conformado en todas sus áreas. En términos generales, el factor limitante de cualquier proceso de conformado es la aparición de una inestabilidad plástica. Para evaluar las posibilidades de aparición de inestabilidades plásticas se están empleando diferentes criterios capaces de predecir y reproducir el proceso físico de dichas inestabilidades (ver Parte I de este trabajo).

Cuando un material metálico se conforma en caliente, a una temperatura, $T$, adecuada, se producen de forma simultánea un endurecimiento por deformación, como consecuencia de la presencia de obstáculos al movimiento de dislocaciones, y un ablandamiento del material deformado por procesos de restauración. Es decir, compiten simultáneamente dos mecanismos antagónicos, de creación y eliminación de defectos cristalinos. Generalmente, se alcanza un equilibrio en aquella competición y se logra un régimen de equilibrio tal, a partir de determinada deformación que, a una tensión constante, el material se deforma plásticamente sin endurecerse.

Así, en un proceso industrial de conformado, un material puede deformarse según varios mecanismos de deformación. En un cierto intervalo de temperatura y velocidad de deformación, sólo actuará uno de los posibles mecanismos de deformación ya que, siendo independientes entre sí, todos ellos actúan simultáneamente y el más rápido será el que controle la fluencia. Un modo de determinar el proceso que controla la fluencia es mediante la utilización de los mapas de procesado ${ }^{[7-15]}$. Estos mapas se construyen usando ecuaciones que relacionan la tensión de fluencia con la temperatura, la velocidad de deformación y la estructura y permiten predecir los mecanismos de deformación controladores a diferentes temperaturas y velocidades de deformación. De esta manera, se pueden definir, en los mapas de procesado, los dominios más "seguros" para el conformado, evitando inestabilidades plásticas y consiguiendo la máxima eficiencia energética.

En esta parte del trabajo se lleva a cabo una caracterización de la fluencia plástica de un acero microaleado de medio carbono mediante un estudio comparativo entre dos tipos de mapas de procesado construidos usando criterios termodinámicos continuos y criterios fenomenológicos.

\section{MÉTODO EXPERIMENTAL}

Como aleación de estudio se escogió un acero comercial microaleado con contenido medio de carbono, destinado al sector de forja de componentes de automoción. Los elementos de aleación son vanadio, titanio y aluminio y su composición química se indica en la tabla I.

Para estudiar el comportamiento a fluencia del acero estudiado se realizaron ensayos de compresión uniaxial a velocidades de deformación verdadera, $\dot{\varepsilon}$, constantes. Los ensayos se efectuaron a temperaturas que oscilaron entre 1.150 y $900^{\circ} \mathrm{C}$, a incrementos de $50^{\circ} \mathrm{C}$ y en un intervalo de velocidades de deformación que varió entre $10^{-4}$ y $10 \mathrm{~s}^{-1}$.

Las probetas ensayadas eran cilíndricas (su eje era paralelo al de laminación) de 11,4 mm de altura y 7,6 $\mathrm{mm}$ de radio. Con estas dimensiones se conseguía minimizar los, prácticamente inevitables, problemas de abarrilamiento y pandeo. Como se puede ver en la

Tabla I. Composición química del acero estudiado (\% en peso)

Table I. Chemical composition of studied steel (weight \%)

\begin{tabular}{cccccccccc}
\hline Acero & $\% \mathrm{C}$ & $\% \mathrm{Mn}$ & $\% \mathrm{Si}$ & $\% \mathrm{P}$ & $\% \mathrm{~S}$ & $\% \mathrm{~V}$ & $\% \mathrm{Al}$ & $\% \mathrm{Ti}$ & $\mathbf{N}_{\mathrm{ppm}}$ \\
\hline $\mathrm{V}$ & 0,34 & 1,52 & 0,72 & 0,025 & 0,025 & 0,083 & 0,0145 & 0,018 & 0,0114 \\
\hline
\end{tabular}




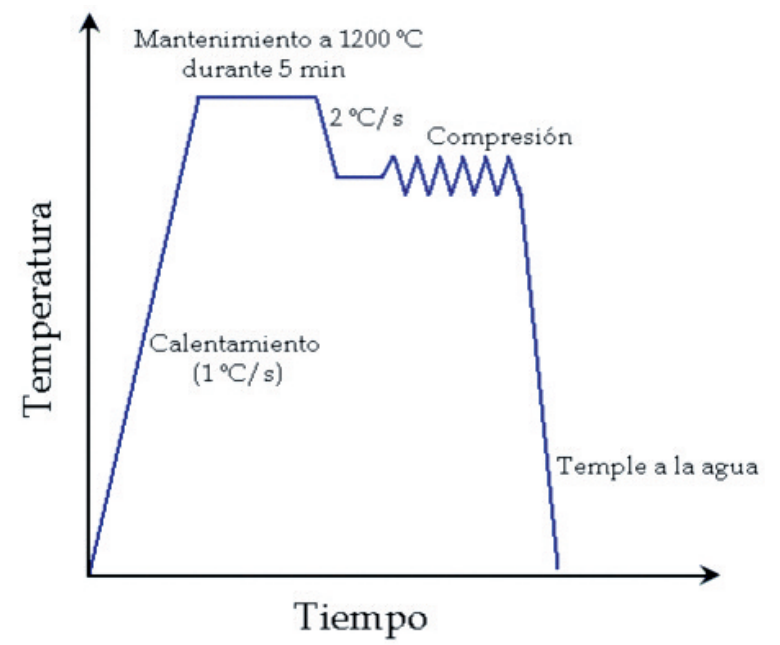

Figura 1. Procedimiento experimental para los ensayos de compresión en caliente.

Figure 1. Experimental procedure for hot compression tests.

figura 1, las probetas se austenizaban durante $5 \mathrm{~min}$ a $1.200^{\circ} \mathrm{C}$ (la velocidad de calentamiento fue de aproximadamente $1^{\circ} \mathrm{C} / \mathrm{s}$ ) y, entonces, se enfriaban hasta la temperatura de ensayo a una velocidad de enfriamiento de $2{ }^{\circ} \mathrm{C} / \mathrm{s}$, aproximadamente. Acabado el ensayo las probetas eran templadas tan rápido como se podía para el posterior análisis del tamaño de grano.

El examen de las microestructuras y la determinación del tamaño de grano austenítico se efectuó mediante microscopía óptica y análisis de imagen sobre probetas templadas en agua una vez acabado el ensayo. Tras desbaste y pulido las muestras se sometieron a ataque químico para revelar los bordes de grano austenítico. Debe notarse que la microestructura inicial es idéntica para todas las probetas ensayadas (tamaño de grano, fracción de volumen de precipitados).

\section{RESULTADOS Y DISCUSIÓN}

\subsection{Curvas de fluencia}

En la figura 2 se representan las curvas de tensión verdadera - deformación verdadera, en función de la temperatura y velocidad de deformación del acero estudiado. Las características principales de las curvas
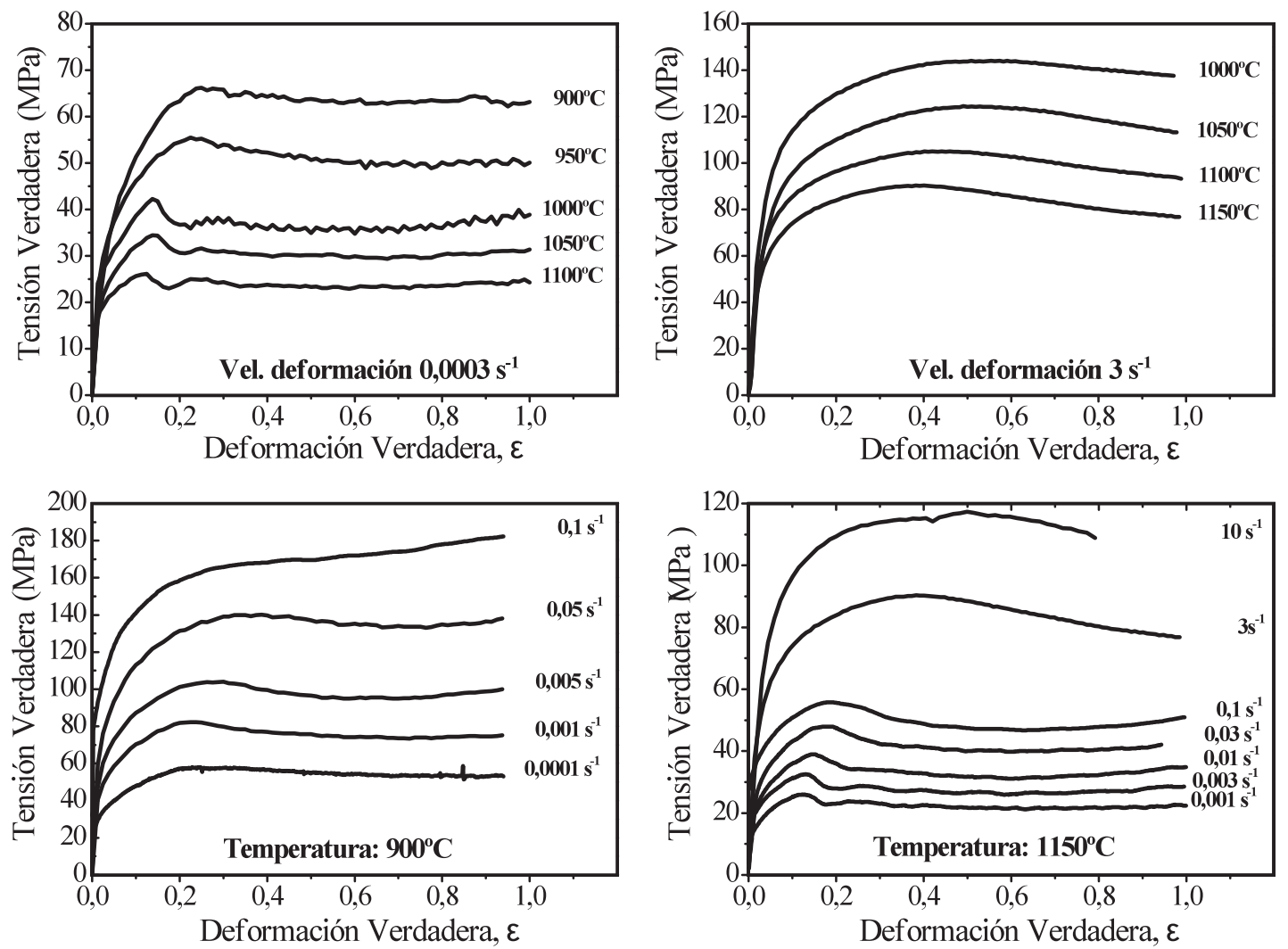

Figura 2. Curvas de fluencia del acero estudiado a varias velocidades de deformación y diferentes temperaturas.

Figure 2. Flow curves of studied steel at various strain rates and different temperatures. 
de fluencia son similares para todas las condiciones experimentales y tienen la tendencia habitualmente observada en los materiales que sufren restauración dinámica (de aquí, en adelante, DRV) y recristalización dinámica (de aquí en adelante, DRX): la tensión aumenta muy rápidamente (endurecimiento por deformación del material) hasta alcanzar un máximo, después del cual, la tensión se mantiene o decrece de manera monótona o con oscilaciones. En algunas situaciones (a $\dot{\varepsilon}$ altas y $T$ bajas), apenas se apreció ablandamiento posterior a la tensión máxima, poniéndose de relieve que el único mecanismo de ablandamiento actuante, entonces, era la DRV. Por otra parte, se observó que, a $T$ constante, la tensión máxima, $\sigma_{p}$, y su deformación asociada, $\varepsilon_{p}$, aumentaban con $\varepsilon$. También, se constató que $\sigma_{p}$ y $\varepsilon_{p}$ disminuyen cuando $T$ aumenta y $\dot{\varepsilon}$ se mantiene constante. En otras palabras, y por lo que a las características mecánicas se refiere, el efecto de disminuir $T$ es equivalente al de aumentar, en un cierto rango de temperaturas $^{[16 \text { y }}{ }^{17]}$. Debe notarse, también, que a medida que va aumentando la temperatura las curvas de fluencia se hacen más sensibles a la velocidad de deformación.

Con objeto de estudiar el efecto de la temperatura y de la velocidad de deformación sobre la tensión de fluencia, se representaron las variaciones de la tensión máxima, $\sigma_{t}$, y de la tensión de estado estable, $\sigma_{s s}$, con $\dot{\varepsilon}$ y con $T$ tal y como se indica en la figura 3. Se observa que, a temperaturas bajas, el comportamiento a fluencia del acero estudiado es, cada vez, más difícil produciendo valores de tensión muy altos. Este efecto se atribuye a que el comportamiento a fluencia del acero estudiado muestra una alta sensibilidad a la temperatura, a temperaturas donde la difusión es lenta, y una baja sensibilidad cuando la difusión contribuye al conformado en caliente. En cuanto al efecto de la velocidad de deformación sobre la tensión de fluencia (Fig. 4), puede apreciarse un progresivo aumento de la tensión de fluencia con la velocidad de deformación para temperaturas entre 900 y $1.150^{\circ} \mathrm{C}$. Esta evolución puede interpretarse en
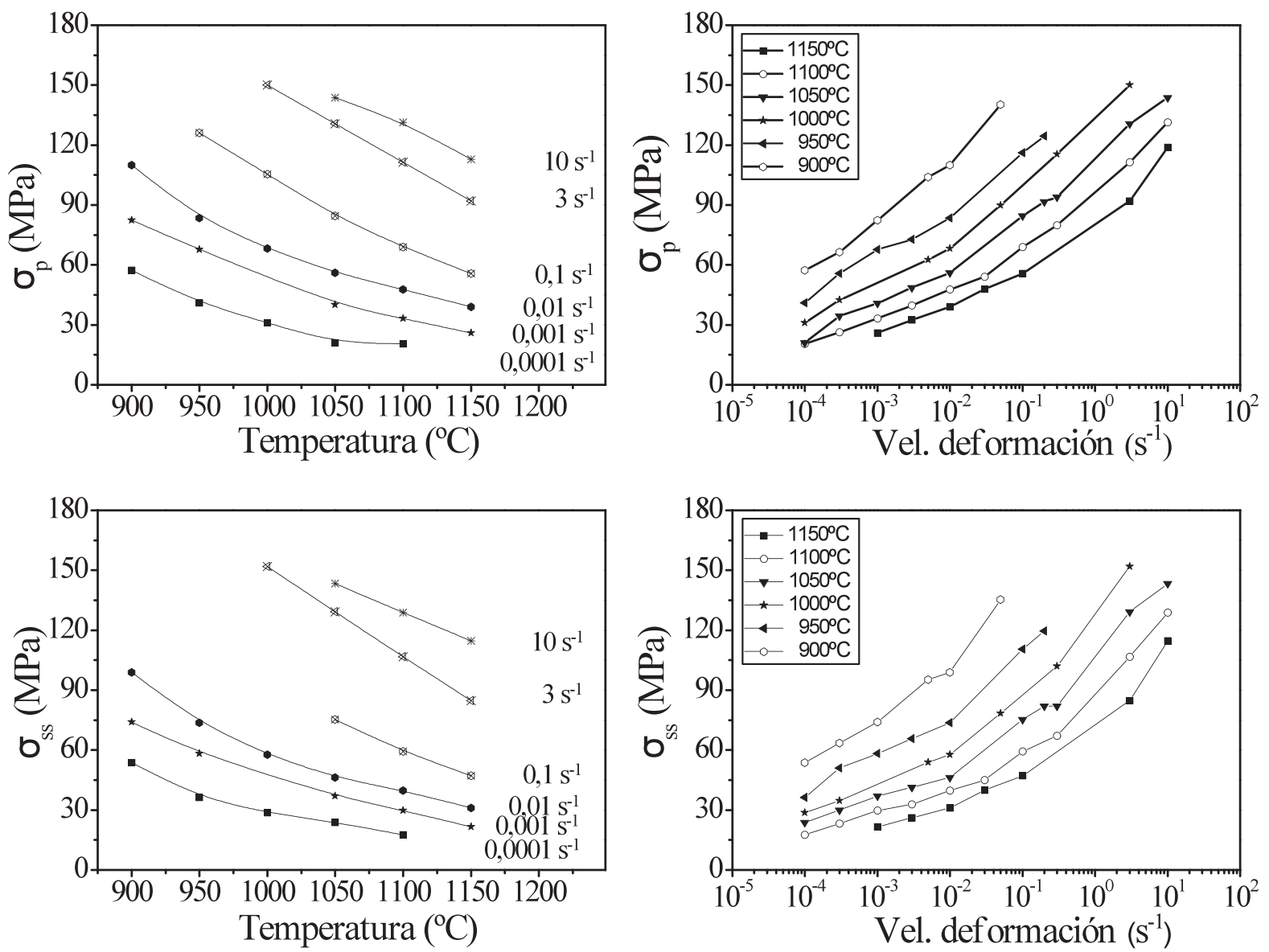

Figura 3. Evolución de la tensión de pico $\sigma_{\mathrm{p}}$ y la tensión de estado estable $\sigma_{\mathrm{ss}}$ con la velocidad de deformación y la temperatura.

Figure 3. Evolution of peak stress $\sigma_{p}$ and steady state stress $\sigma_{s s}$ with strain rate and temperature. 

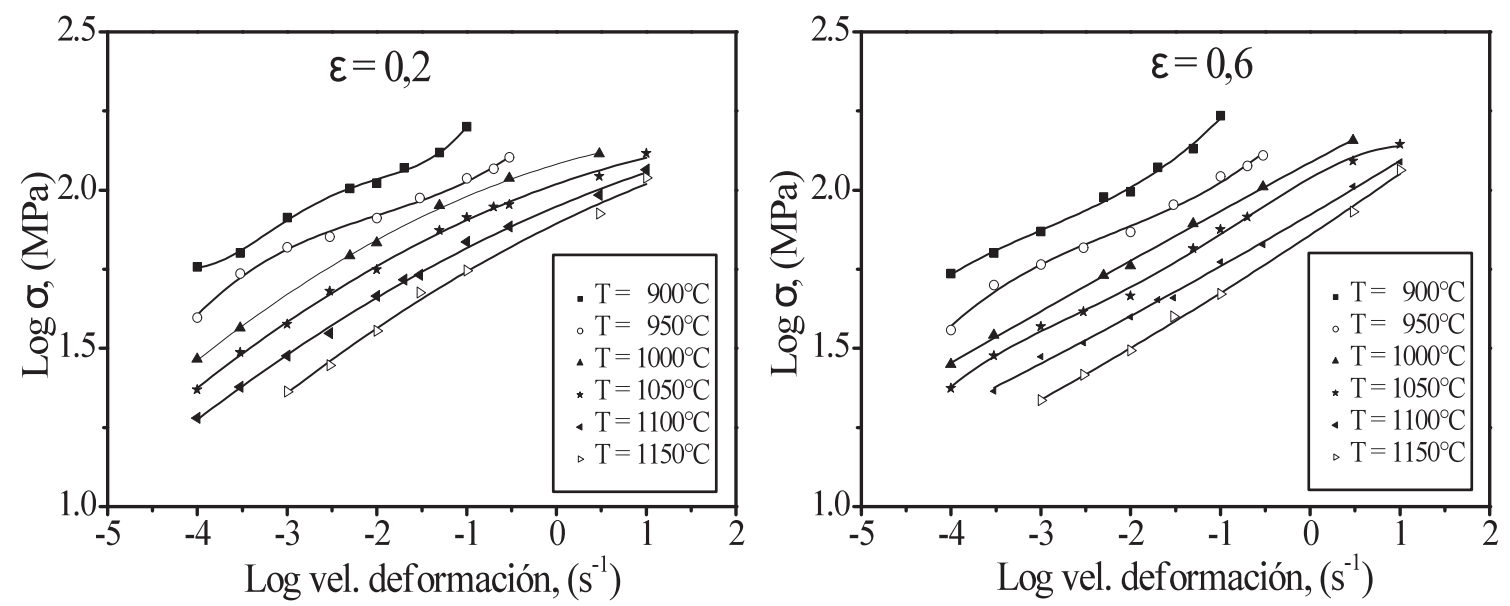

Figura 4. Evolución de la tensión de fluencia con la velocidad de deformación a deformaciones de 0,2 y 0,6 .

Figure 4. Evolution of flow stress with strain rate for strains of 0.2 and 0.6 .

términos de la velocidad de generación de dislocaciones $^{[18]}$; la velocidad de endurecimiento por deformación en el acero estudiado aumenta con la velocidad de deformación desde $10^{-4} \mathrm{~s}^{-1}$ a $10 \mathrm{~s}^{-1}$.

Para determinar el parámetro de sensibilidad a la velocidad de deformación, $m$, del material estudiado, las curvas $\log (\mathrm{s})$ frente a $\log (\dot{\varepsilon})$ se ajustaron a una ecuación polinomial y el parámetro $m$ se determinó a partir de la pendiente de las curvas, a varias

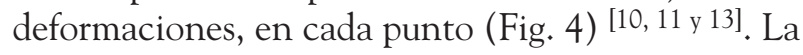
evolución de $m$ con $T$ y $\dot{\varepsilon}$ a deformaciones de 0,2 y 0,6 se muestra en la figura 5 . El parámetro $m$ varía, notablemente, con la deformación; esto quiere decir que la deformación afecta sensiblemente al proceso de conformado, lo cual es lógico ya que diferentes mecanismos de deformación actúan a distintas combinaciones de $T$ y $\dot{\varepsilon}$. A temperaturas superiores a $950^{\circ} \mathrm{C}, m$ aumenta con la temperatura y disminuye con la velocidad de deformación, a bajas deformaciones $(\varepsilon=0,2)$. Sin embargo, a altas deformaciones, el material se hace menos sensible a las variaciones de $T$ y $\dot{\varepsilon}$. Para mejor visualización, la variación de $m$ con $T$ y $\dot{\varepsilon}$ se representa como mapas de contorno de iso-m. Los mapas obtenidos a deformaciones de 0,2 y 0,6 se muestran en la figura 6. La interpretación de estos mapas se basa en el hecho de que el material presenta algún tipo de instabilidad plástica cuando el parámetro $m$ toma valores negativos ${ }^{[19]}$; además, es ampliamente reconocido que altos valores de $m$ reducen la posibilidad de aparición de la localización de fluencia y conducen a un máximo de ductilidad en el material.

El mapa de sensibilidad a la velocidad de deformación correspondiente a la deformación de 0,2, exhibe dos dominios interesantes. El primero, caracterizado por un máximo de sensibilidad a la velocidad de deformación de, aproximadamente, 0,23 produciéndose a $1.025^{\circ} \mathrm{C}$ y $10^{-4} \mathrm{~s}^{-1}$. La observación de las curvas de fluencia (Fig. 2) muestra que a $\varepsilon=0,2$ sólo tiene lugar la recristalización dinámica cíclica (de aquí en adelante, CDRX) cuando la deformación se efectúa a bajas y $T$ altas. Esta interpretación se confirma por el crecimiento de grano observado en este dominio respecto al tamaño inicial ${ }^{[10,11,13 \text { y } 20]}$ y es bien conocido y aceptado que la CDRX está asociada a tal crecimiento ${ }^{[16-18]}$.

En el segundo dominio, centrado en $975^{\circ} \mathrm{C}$ y $10 \mathrm{~s}^{-1}$, la sensibilidad a la velocidad de deformación alcanza sus valores más bajos (aproximadamente, $0,05)$. Ello supondría que este dominio, debido a los bajos valores de $m$, puede representar la tendencia que tiene el material estudiado a desarrollar alguna manifestación de inestabilidad de fluencia y baja conformabilidad (grietas, bandas de cizalladura, etc. $)^{[21-}$ 24]. Es importante hacer notar que este mismo dominio adquiere altos valores de $m$ en el mapa correspondiente a la deformación de 0,6.

A $\varepsilon=0,6$, que es, aproximadamente, la deformación correspondiente a fluencia de estado estable, el mapa del parámetro $m$ cambia su aspecto. El análisis de este mapa sugiere que los dominios donde $m$ alcanza sus valores máximos corresponde a dominios de DRX de pico simple y DRV, tal como se había detectado en los mapas de disipación de energía desarrollados en los trabajos de los presentes autores ${ }^{[10,11 \text { y } 13]}$ y los resultados no difieren sensiblemente de los encontrados utilizando el DMM (modelo dinámico de materiales). En consecuencia, el primer dominio de conformabilidad óptima, correspondiente al dominio de DRV, se encuentra 

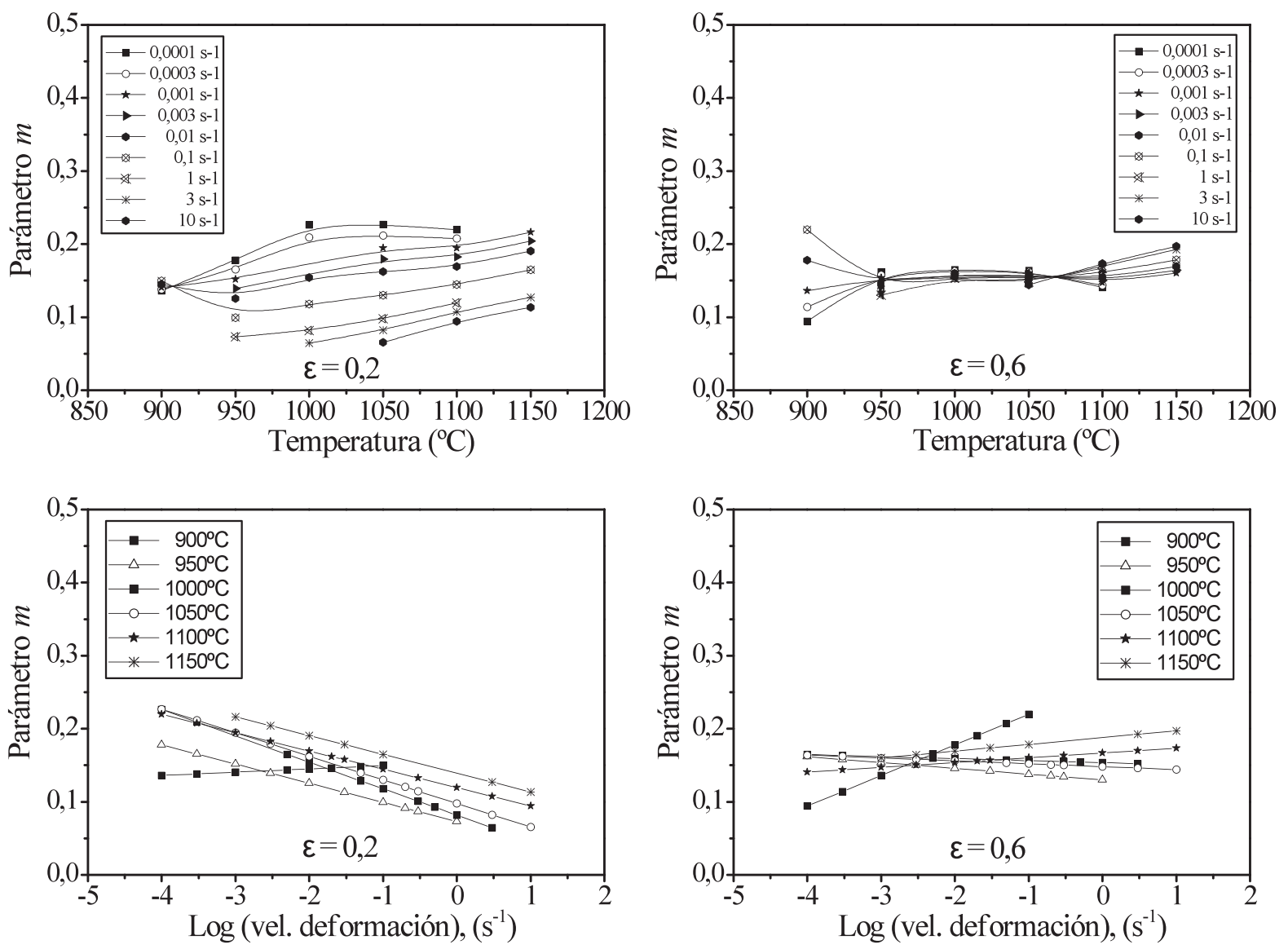

Figura 5. Evolución del parámetro de sensibilidad a la velocidad de deformación $(m)$ con la temperatura y la velocidad de deformación a deformaciones de 0,2 y 0,6.

Figure 5. Evolution of strain rate sensitivity parameter $(m)$ with temperature and strain rate for strains of 0.2 and 0.6 .

(a)

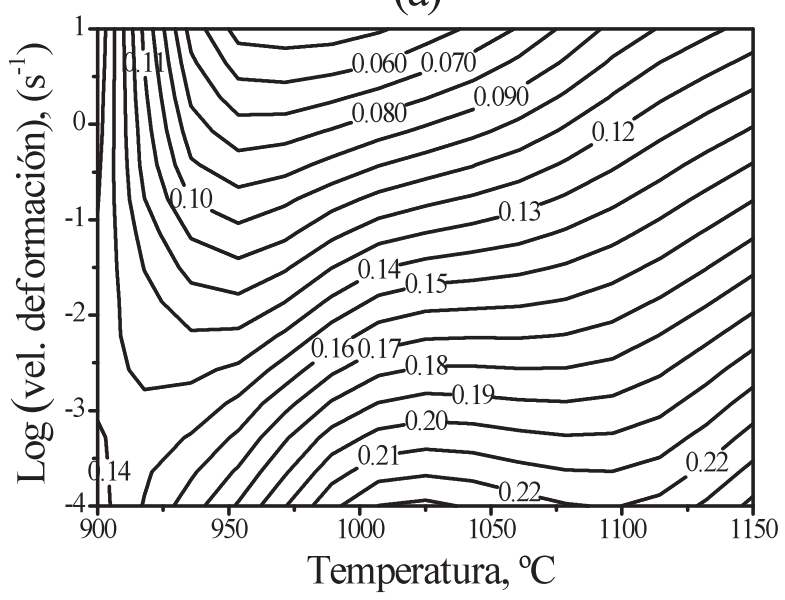

(b)

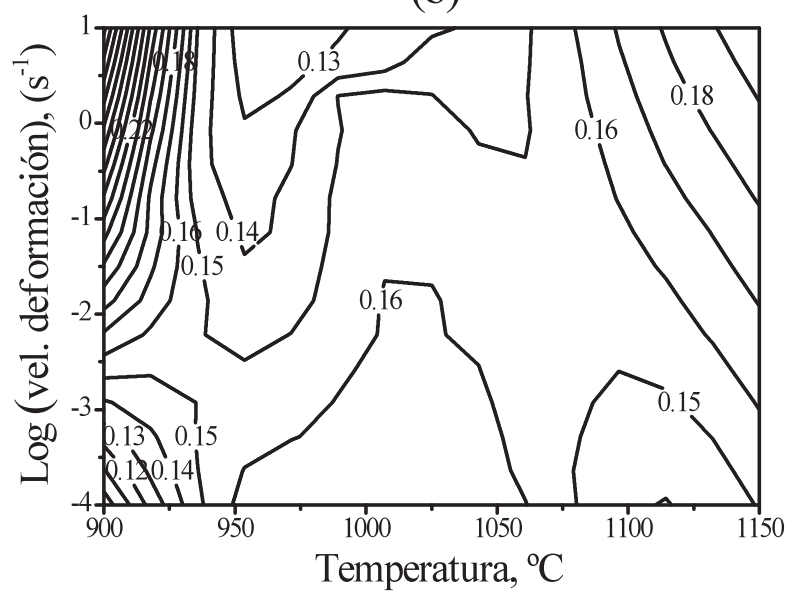

Figura 6. Mapas de contorno del parámetro de sensibilidad a la velocidad de deformación $(m)$ a deformaciones de (a) $\varepsilon=0,2$ y (b) $\varepsilon=0,6$.

Figure 6. Strain rate sensitivity contour maps at: (a) $\varepsilon=0.2$ and (b) $\varepsilon=0.6$. 
extendido sobre el rango de temperaturas de 900 a $950^{\circ} \mathrm{C}$ y el rango de velocidades de deformación de 0,003 a $10 \mathrm{~s}^{-1}$, con un valor máximo de $m$ de, aproximadamente, 0,30. El segundo dominio de conformabilidad óptima, correspondiente al dominio de DRX, centrado en $1.150^{\circ} \mathrm{C}_{\text {y }} 10 \mathrm{~s}^{-1}$ con un valor de $m$ de, aproximadamente, 0,20 . Esta interpretación se confirma mediante el análisis de los mapas de contorno iso-tamaño de grano recristalizado y los mapas de contorno representando las variaciones del grado de ablandamiento en función de la temperatura y velocidad de deformación dados en los trabajos de estos mismos recien citados autores $^{[10,11,13]}$.

\subsection{Mapas de inestabilidad}

Los mapas de inestabilidad construidos en base al criterio dado en la ecuación (74.I), a deformaciones de 0,2 y 0,6 , se muestran en la figura 7 . Estos mapas indican que las regiones donde se predice la aparición de los procesos de DRV y DRX son las regiones más estables y el parámetro $\xi_{p}(T, \dot{\varepsilon})$ (la letra $P$ hace referencia a Prasad, uno de los autores del DMM) toma valores positivos y alcanza sus valores máximos, lo cual es consistente con que tales regiones son las más seguras de los mapas de procesado.

En el mapa de inestabilidad correspondiente a $\varepsilon=0,2$, exhibe un dominio con valores negativos

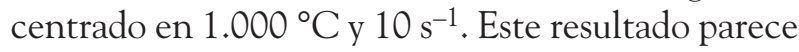
indicar que el dominio en cuestión representa zonas de baja conformabilidad debido a la aparición de inestabilidades plásticas ${ }^{[25-27]}$. Este eventual dominio de inestabilidad plástica coincide con el dominio previsto en los mapas de contorno del parámetro, $m$ (Fig. 6 a)). Sin embargo, puesto que las observaciones microestructurales mediante microscopía óptica y electrónica de barrido ${ }^{[10]}$ no evidenciaron ninguna inestabilidad plástica, puede argumentarse que la inestabilidad que predicen estos mapas se debe al efecto térmico o calentamiento adiabático, el cual no fue corregido en las curvas experimentales. También, cabría pensar en la posibilidad de que la supuesta manifestación de inestabilidad no es lo suficientemente intensa como para mantenerse durante un cierto tiempo debido a la relativamente baja deformación impuesta $(\varepsilon=0,2)$. No obstante, dicha manifestación no puede atribuirse a la aparición de bandas de cizalladura ya que al recristalizarse el material estudiado se producen cambios estructurales que hacen desaparecer a estas bandas. A altas deformaciones (Fig. 7 b)), este dominio se ha movido y se ha se ha centrado en $955^{\circ} \mathrm{C}$ y $0,5 \mathrm{~s}^{-1}$ pero, ahora, teniendo valores positivos de $\xi_{\mathrm{p}}$. Como se ha interpretado en el mapa de sensibilidad a la velocidad de deformación de la figura $6 \mathrm{~b}$ ), este dominio es a evitar, a la hora de conformar en caliente el material objeto de este estudio, debido a su baja conformabilidad.

En la figura 8 se han representado, para las deformaciones $\varepsilon=0,2$ y $\varepsilon=0,6$, los mapas de inestabilidad obtenidos en base al criterio de inestabilidad establecido en la ecuación (85.I) del DMM modificado, (a)

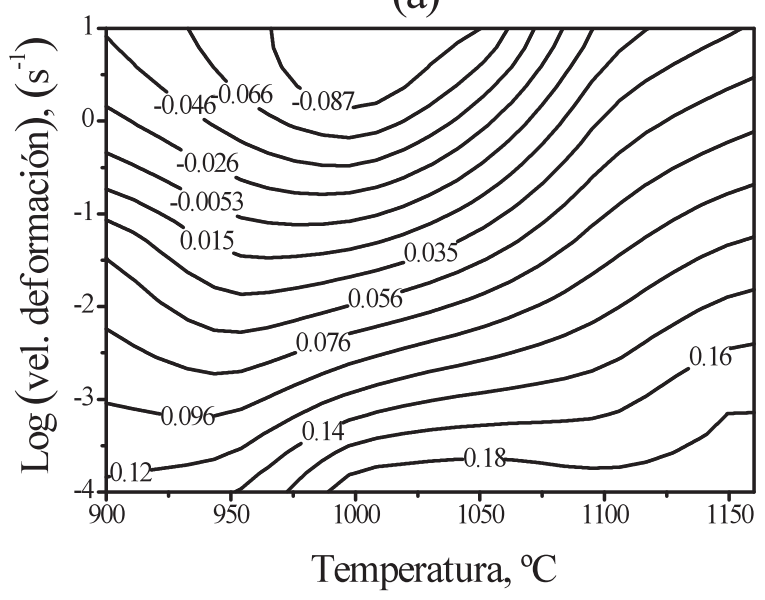

(b)

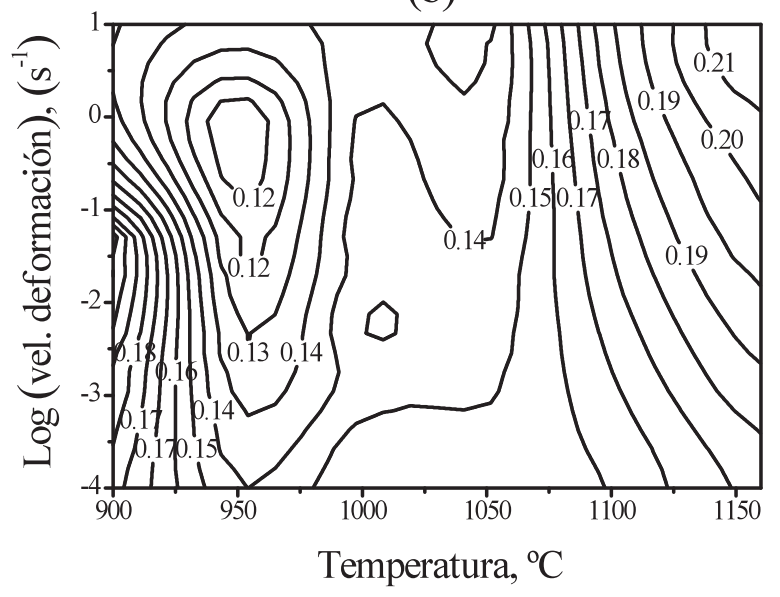

Figura 7. Mapas de contorno del parámetro de inestabilidad $\xi_{P}(T, \dot{\varepsilon})$ obtenido a partir de la ecuación (39.II) a deformaciones de (a) $\varepsilon=0,2$ y (b) $\varepsilon=0,6$.

Figure 7. Maps of instability parameter $\xi_{P}(T, \dot{\varepsilon})$ obtained from Equation (39.II) at: (a) $\varepsilon=0.2$ and (b) $\varepsilon=0.6$. 
(a)

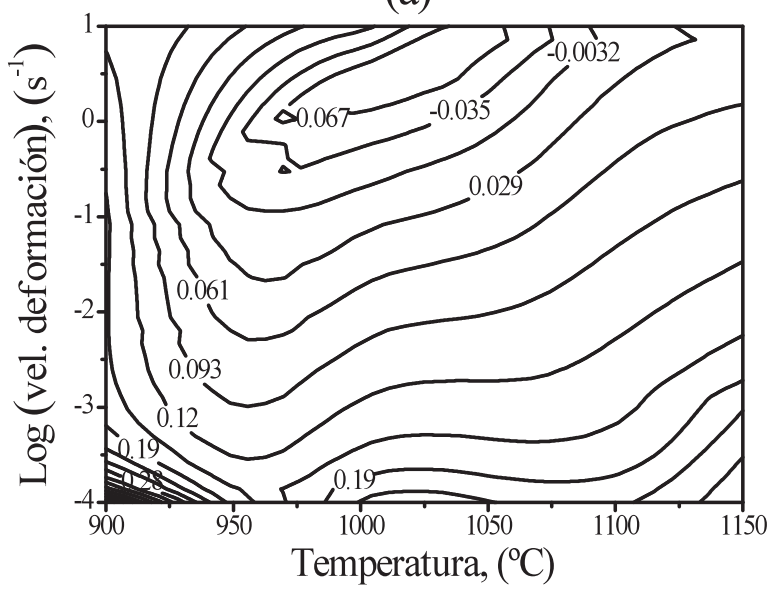

(b)

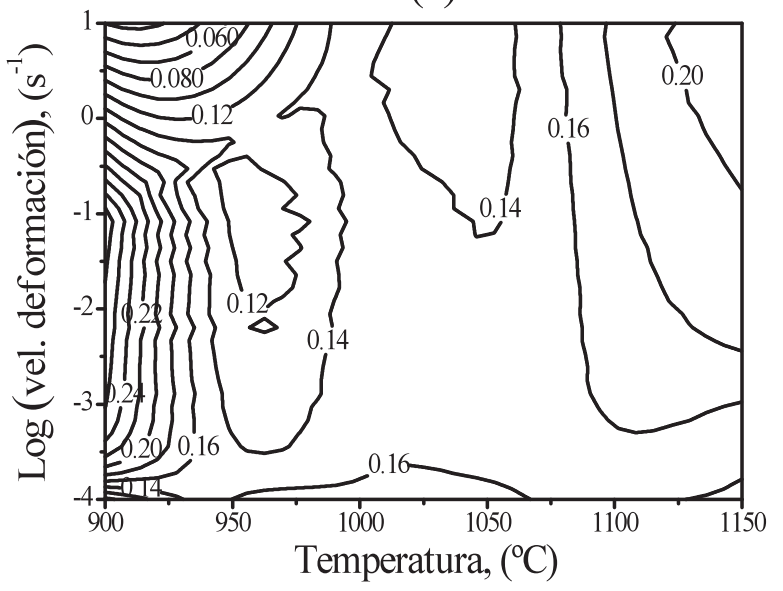

Figura 8. Mapas de contorno del parámetro de inestabilidad $\xi_{N}(T, \dot{\varepsilon})$ obtenido a partir de la ecuación (51.II) a deformaciones de (a) $\varepsilon=0,2$ y (b) $\varepsilon=0,6$.

Figure 8. Maps of instability parameter $\xi_{N}(T, \dot{\varepsilon})$ obtained from Equation (51.II) at: (a) $\varepsilon=0.2$ and (b) $\varepsilon=0.6$.

desarrollado por Narayana et al. (véase Parte I). Los números de los contornos indican los valores del parámetro de inestabilidad, $\xi_{N}(T, \dot{\varepsilon})$ (la letra $N$ hace referencia a Narayana). Tal como puede observarse en la figura 8, las características generales de los dos mapas varían, considerablemente, con la deformación $\mathrm{y}$, a medida que aumenta, van apareciendo nuevos dominios.

El mapa de inestabilidad del parámetro $\xi_{N}(T, \dot{\varepsilon})$ a $\varepsilon=0,2$, puede dividirse en tres dominios. El primer dominio está centrado en $900^{\circ} \mathrm{C}$ y $10^{-4} \mathrm{~s}^{-1}$, con un valor máximo de $\xi_{N}(T, \dot{\varepsilon})$ de, aproximadamente, 0,5 . Las curvas de fluencia obtenidas con diferentes combinaciones de $T$ y $\dot{\varepsilon}$, en este dominio, muestran claramente que el único mecanismo que puede tener lugar a esta deformación es la DRV. El segundo dominio, centrado en $1.025^{\circ} \mathrm{C}$ y $10^{-4} \mathrm{~s}^{-1}$, con un pico del parámetro $\xi_{N}(T, \dot{\varepsilon})$ de 0,2 , puede interpretarse, al igual que en el mapa del parámetro $m$ a $\varepsilon=0,2$, como un dominio representativo de la CDRX. El ter-

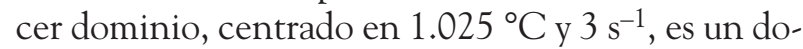
minio caracterizado por valores negativos de $\xi_{N}$ ( $T$, $\dot{\varepsilon})$ y puede identificarse con los dominios de inestabilidad previstos en las figuras 6 a) y 7 a). En general, existe un buen acuerdo entre los dominios de los tres mapas (Figs. 6 a), 7 a) y 8 a)) y la diferencia observada en la posición del dominio se debe, principalmente, a los métodos de cálculo de los distintos parámetros $m, \xi_{P}(T, \dot{\varepsilon})$ y $\xi_{N}(T, \dot{\varepsilon})$.

A altas deformaciones $(\varepsilon=0,6)$ el mapa de contorno de $\xi_{N}(T, \dot{\varepsilon})$ exhibe un nuevo dominio centrado

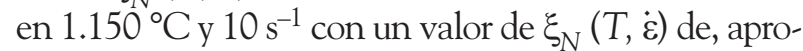
ximadamente, 0,2. Este dominio puede atribuirse, fácilmente, a la DRX de pico simple observado en los mapas de las figuras 6 b) y 7 b) y situado en la misma posición. El dominio de la DRV observado en el mapa correspondiente a $\varepsilon=0,2$ se ha movido hacia moderadas velocidades de deformación (centrado en $900^{\circ} \mathrm{C}$ y $10^{-2} \mathrm{~s}^{-1}$ ). También, este dominio coincide con el dominio de la DRV identificado en los mapas de las figuras 6 b) y 7 b), con una pequeña diferencia en la posición del dominio, en cada mapa. El dominio con valores negativos de $\xi_{N}(T, \dot{\varepsilon})$ ha desaparecido completamente y el valor mínimo de $\xi_{N}(T, \dot{\varepsilon})$ observado en la figura 8 b) es 0,05 , que aparece a $910^{\circ} \mathrm{C}$ y $10 \mathrm{~s}^{-1}$. Para asegurar que el proceso de conformado en caliente pueda llevarse a cabo con total seguridad, este dominio, caracterizado por valores mínimos de $\xi_{N}(T, \dot{\varepsilon})$, debe evitarse durante el conformado del material estudiado.

Con objeto de confirmar el buen acuerdo y la similitud entre los resultados del DMM y del DMM modificado, se ha representado, en la figura 9, en un mismo gráfico, la evolución, con la velocidad de deformación a $T=950{ }^{\circ} \mathrm{C}$ y $\mathrm{T}=1.150{ }^{\circ} \mathrm{C}$ para las deformaciones $\varepsilon=0,2$ y $\varepsilon=0,6$, de la eficiencia de disipación de energía definida en el DMM; $\eta=\frac{2 m}{m+1}$ (véase Parte I) y la definida en el DMM modificado y expresada en la ecuación (82.I). Puede observarse que, a $\varepsilon=0,2$, la diferencia entre los valores de la eficiencia del DMM y del DMM modificado es mínima y esta diferencia ha desaparecido, casi por completo, a $\varepsilon=0,6$.

A pesar de las diferencias existentes en los métodos de cálculo utilizados para establecer los tres criterios 

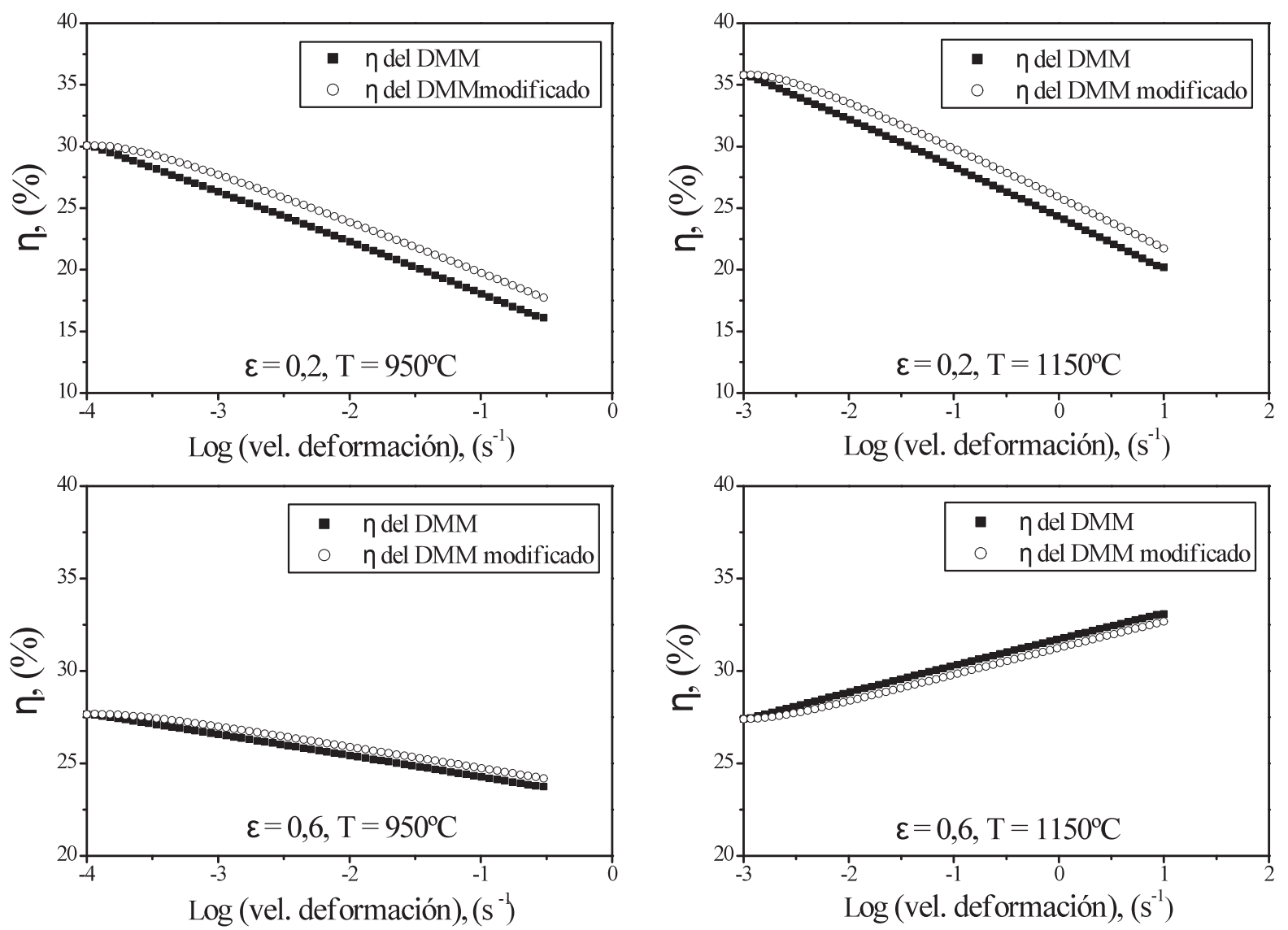

Figura 9. Comparación entre los valores de la eficiencia de disipación de energía definida en el DMM y en el DMM modificado.

\section{Figure 9. Comparison of values of efficiency of power dissipation defined in DMM and modified DMM.}

de estabilidad, la comparación entre los mapas obtenidos mediante estos criterios, es decir, $0<m<1$, $\xi_{\mathrm{N}}(\dot{\varepsilon}, T),>0$ y $\xi_{\mathrm{P}}(\dot{\varepsilon}, T),>0$, pone de manifiesto el buen acuerdo que existe entre los resultados; y el comportamiento de estabilidad del material estudiado en la presente investigación es sims ilar para los tres criterios. El principal dominio de estabilidad, correspondiente a las condiciones óptimas de conformado, se ha observado a altas temperaturas y altas velocidades de deformación (dominio de la DRX). El segundo dominio estable, correspondiente a la DRV, se ha detectado en la zona extendida de moderadas hacia altas $\dot{\varepsilon}$ y $T=900^{\circ} \mathrm{C}$. Para validar, microestructuralmente, las interpretaciones realizadas en el presente trabajo sería interesante llevar a cabo un estudio más exhaustivo de la microestructura del acero empleado, especialmente, mediante la microscopía electrónica de transmisión y microscopía electrónica de barrido, para ver si es posible identificar alguna inestabilidad plástica. No cabe olvidar que las probetas fueron deformadas hasta la deformación, $\varepsilon=1 \mathrm{y}$, una vez acabado el ensayo, se templaron en agua para congelar la microestructura. Lo ideal sería templar las probetas a diferentes deformaciones del proceso de conformado para poder seguir la evolución microestructural, lo que no fue posible en este trabajo, por lo que no se hizo un examen microestructural de las probetas a deformación 0,2 y 0,6 sino a deformación $\varepsilon=1$.

Los mapas de inestabilidad construidos en base al criterio fenomenológico desarrollado por Semaitin, Jonas y Lahoti ${ }^{[21,31}$ y 32] y a la variación del al parámetro $\xi_{S}(T, \dot{\varepsilon})$ (la letra $S$ hace referencia a Semiatin, uno de los autores del criterio fenomenológico) obtenido a partir de la ecuación (36.I), desarrollada en la Parte I del presente trabajo, se muestran la figura 10 , para las deformaciones $\varepsilon=0,2$ y $\varepsilon=0,6$. De acuerdo con el criterio fenomenológico, en procesos de conformado en caliente, los materiales exhiben una localización significativa de la fluencia cuando los dominios de los mapas del parámetro $\xi_{S}(T$, $\dot{\varepsilon})$ toman valores negativos. Puede observarse, en la 
(a)

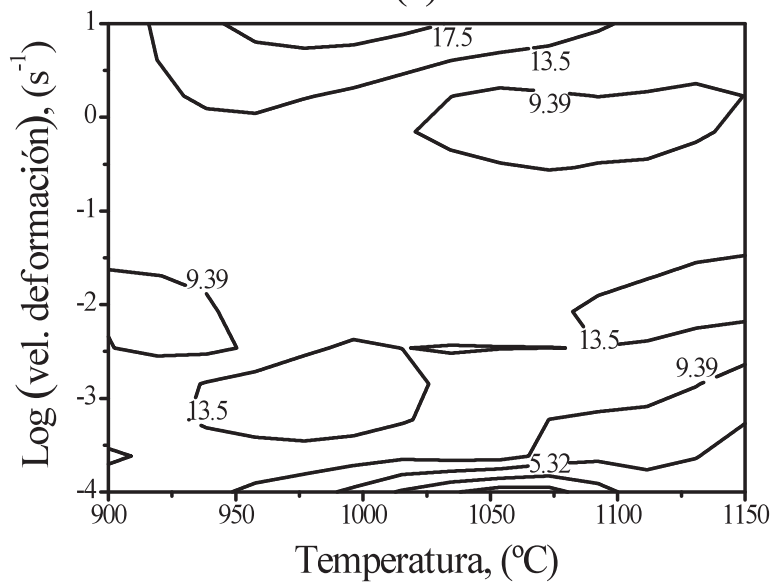

(b)

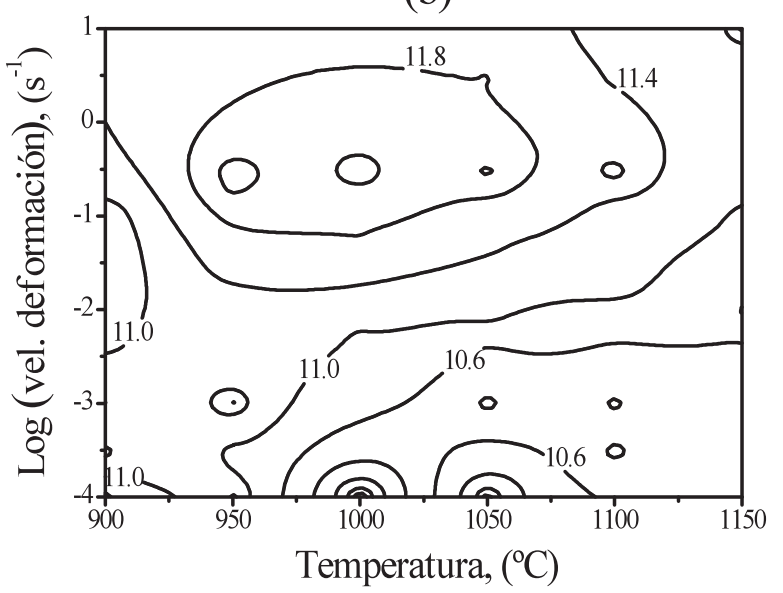

Figura 10. Mapas de contorno del parámetro de inestabilidad $\xi_{S}(T, \dot{\varepsilon})$ obtenido a partir de la ecuación (30.I) a deformaciones de (a) $\varepsilon=0,2$ y (b) $\varepsilon=0,6$.

Figure 10. Maps of instability parameter $\xi_{s}(T, \dot{\varepsilon})$ obtained from Equation (30.I) at: (a) $\varepsilon=0.2$ and (b) $\varepsilon=0.6$.

figura 10 a), que la fluencia estable aparece en la zona centrada en $980^{\circ} \mathrm{C}_{\text {y }} 10 \mathrm{~s}^{-1}$, mientras que la zona de la fluencia inestable está centrada en $1.065^{\circ} \mathrm{C}$ y $10^{-4} \mathrm{~s}^{-1}$, con un valor mínimo de $\xi_{S}(T, \dot{\varepsilon})$ de, aproximadamente, $-3,3$. Estos resultados contradicen los encontrados en las figuras 6 a), 7 a) y 8 a). A $\varepsilon=0,6$, el mapa de inestabilidad exhibe dos dominios de fluencia estable: el primero, extendido sobre el rango de temperaturas de $900 \mathrm{a} 1.125^{\circ} \mathrm{C}$ y sobre el rango de velocidades de deformación de $10^{-2}$ a $10 \mathrm{~s}^{-1}$, con un valor máximo de $\xi_{S}(T, \dot{\varepsilon})$ de, aproximadamente, 12,2 (Fig. 10 b)). El segundo dominio se divide en dos pequeños dominios centrados en $1.000^{\circ}$ $\mathrm{C}, 10^{-4} \mathrm{~s}^{-1}$ y $1050^{\circ} \mathrm{C}, 10^{-4} \mathrm{~s}^{-1}$, respectivamente (Fig. 10 b)). Estos dos dominios pueden asociarse con la CDRX de acuerdo con las curvas de fluencia obtenidas con diferentes combinaciones de $T$ y $\dot{\varepsilon}$, en estos dos dominios.

Para el material estudiado en este trabajo, parece ser que el criterio fenomenológico de inestabilidad no proporciona suficiente información para predecir los cambios microestructurales susceptibles de producirse durante el proceso de conformado en caliente. Además, el carácter empírico de este criterio hace difícil la interpretación de sus mapas de inestabilidad. Cabe señalar que el valor constante de 5, expresado en la ecuación (36.I). se ha utilizado para detectar la localización de la fluencia durante la deformación en caliente del titanio y sus aleaciones ${ }^{[21,}$ 31 y 32]. Para otros materiales, este valor constante puede ser diferente y su validez debe ser comprobada por estudios microestructurales exhaustivos.

\section{CONCLUSIONES}

En el presente trabajo, se han utilizado criterios continuos y fenomenológicos para caracterizar la inestabilidad plástica en un acero microaleado de medio del carbono. Del análisis de los resultados obtenidos, las principales conclusiones que se deducen son: - Se ha confirmado que el acero estudiado, no exhibe ningún tipo de manifestación de la inestabilidad plástica durante su conformado en caliente llevado a cabo en un rango de temperaturas de $900^{\circ} \mathrm{C}$ a $1.150^{\circ} \mathrm{C}$ y a diferentes velocidades de deformación que van desde $10^{-4}$ a $10 \mathrm{~s}^{-1}$.

- A pesar de la pequeña diferencia encontrada en la posición de los dominios estables, se ha observado un buen acuerdo entre los diferentes mapas de inestabilidad construidos en base a los criterios continuos.

- Todos los mapas de inestabilidad correspondientes a la deformación $\varepsilon=0,6$ muestran un dominio de DRX que puede ser considerado como el dominio más "seguro" para el conformado en caliente. Este dominio está centrado a $1.150^{\circ} \mathrm{C}$ y $10 \mathrm{~s}^{-1}$. Además, estos mapas han revelado la existencia de un dominio de DRV a bajas temperaturas y moderada velocidad de deformación.

- El dominio de la DRX es muy importante en la optimización de la conformabilidad intrínseca, puesto que reconstituye la microestructura mediante formación y migración de los límites de grano. En consecuencia, este dominio pondrá los límites para el control de los parámetros del proceso de conformado. 
- Para incrementar la productividad, en condiciones industriales, las primeras operaciones de conformado en caliente deben realizarse con una temperatura del orden de $1.150^{\circ} \mathrm{C}$ y con una velocidad de deformación de $10 \mathrm{~s}^{-1}$ (dominio DRX), mientras que las últimas operaciones deben efectuarse en el dominio DRV (centrado a $900^{\circ} \mathrm{C}$ y $0,1 \mathrm{~s}^{-1}$ ). De esta manera, se obtiene una microestructura con un tamaño de grano fino, lo cual mejora considerablemente las propiedades mecánicas del acero estudiado, sobre todo la tenacidad.

- En comparación con el criterio fenomenológico, los criterios termodinámicos continuos predicen con mayor exactitud los cambios microestructurales y son más eficientes para optimizar los parámetros de control del proceso de conformado en caliente del acero estudiado.

\section{Agradecimientos}

Uno de los autores, Anas Al Omar, desea agradecer a la CICYT (España) la financiación económica a través del proyecto DPI2007-63665.

\section{REFERENCIAS}

[1] T. Gladman, Ironmaking Steelmaking 16 (1989) 241-245.

[2] F.B. Pickering, Physical metallurgy and the design of steels, Cap. 4, Ed. Applied Science Publishers, Essex, 1978.

[3] W.C. Leslie, The physical metallurgy of steels, Cap. 6, Ed. McGraw Hill International, Tokyo, Japón, 1982.

[4] C.A. Hernández, S. F. Medina, J. E. Mancilla y V. Blázquez, Rev. Metal. Madrid 28 (1992) 369-382.

[5] S. F. Medina y J. E. Mancilla, ISIJ Int. 36 (1996) 1063-1069.

[6] J. Castellanos, V. Gutiérrez, I. Rieiro, O. A. Ruano y M. Carsí, Rev. Metal. Madrid 45 (2009), doi: 10.3989/revmetalm.0837.

[7] J. Luo, M. Li, W. Yu y H. Li, Mater. Sci. Eng. A 504 (2009) 90-98.

[8] S.V.S. Narayana Murty y B. Nageswara Rao, J. Mater. Process. Technol. 14 (2000) 103-109.

[9] Y.V.R.K. Prasad y S. Sasidhara, Hot Working Guide: A Compendium of Processing Maps, ASM International, Materials Park, Ohio, 1997.
[10] A. Al Omar, Tesis Doctoral, Universidad Politécnica de Cataluña, 1996.

[11] A. Al Omar, J.M. Cabrera y J.M. Prado, Scr. Mater.34 (1996) 1.303-1.308.

[12] A. Al Omar y J.M. Prado, Rev. Metal. Madrid 33 (1997) 89-100.

[13] A. Al Omar, J.M. Cabrera y J.M. Prado, Rev. Metal. Madrid 33 (1997) 153-160.

[14] I. Rieiro, A. Fernández, A. Martínez y M. Carsí, Rev. Metal. Madrid 34 (1998) 355-366.

[15] I. Rieiro, M. Cars> y O.A. Ruano, Mater. Sci. Technol. 25 (2009) 995-1.002.

[16] T. Sakai y J.J. Jonas, Acta Metall. 32 (1984) 189209.

[17] M.J. Luton y C.M. Sellars, Acta Metall. 17 (1969) 1.033-1.043.

[18] H.J. McQueen y J.J. Jonas, Treatise on Materials Science and Technology 6 Academic Press, 1975, pp. 393-493.

[19] F. Montheillet, J. J. Jonas y K.W. Neale, Metall. Trans. A 27 (1996) 232-235.

[20] A. Al Omar, A. Chenaoui, R. Dkiouak, J.M. Cabrera y J.M. Prado, Rev. Metal. Madrid 42 (2006) 103-113.

[21] S.L. Semiatin y J.J. Jonas, Formability and Workability of Metals: Plastic Instability and Flow Localization, ASM, Metals Park, Ohio, 1984.

[22] S. I. Oh, S. L. Semiatin y J. J. Jonas, Metall. Trans. A 23 (1992) 963-975.

[23] E. Rauch, G. R. Canova, J. J. Jonas y S. L. Semiatin, Acta Metall. 33 (1985) 465-476.

[24] P. Dadras y J. F. Thomas, Jr., Res. Mechanica Letters 1 (1981) 97.

[25] J.K. Chakravartty, R. Kapoor, S. Banerjee y Y.V.R.K. Prasad, Mater. J. Nucl. Mater. 362 (2007) 75-86.

[26] Y.V.R.K. Prasad y K.P. Rao, Mater. Sci. Eng. A 391 (2005) 141-150.

[27] P.V. Sivaprasad, S.L. Mannan y Y.V.R.K. Prasad, Mater. Sci. Technol. 20 (2004) 1.545-1.550.

[28] Nho-Kwang Park, Jong-Taek Yeom y YoungSang Na, J. Mater. Process. Technol. 130131(2002) 540-545.

[29] Yi Liu, Rui Hu, Jinshan Li, Hongchao Kou, Hongwei Li, Hui Chang y Hengzhi Fu, J. Mater. Process. Technol. 209 (2009) 4.020-4.026.

[30] K. Muraleedharan, y. V. R. K. Prasad Y V. Singh, Mater. Sci. Eng. A 245 (1998) 88-99.

[31] S. L. Semiatin y G. D. Lahoti, Metall. Trans. A 13 (1982) 275-288.

[32] S. L. Semiatin y G. D. Lahoti, Metall. Trans. A 12 (1981) 1.719-1.728. 